\title{
Habenula and Thalamus Cell Transplants Mediate Different Specific Patterns of Innervation in the Interpeduncular Nucleus
}

\author{
Theresa C. Eckenrode, Marion Murray, and Forrest Haun \\ Department of Anatomy and Neurobiology, Medical College of Pennsylvania, Philadelphia, Pennsylvania 19129
}

Innervation of specific peptidergic and cholinergic compartments of the interpeduncular nucleus (IPN) was investigated using embryonic cell suspension transplants immunoreactive for substance P (SP) and ChAT. In both neonatal and adult host rats, the IPN was first denervated of its normal SP and cholinergic input from the medial habenula by bilateral lesions of the fasciculi retroflexi (FR). In adult hosts, transplants of embryonic habenular cells placed near the denervated IPN mediated a return of the normal pattern of SP staining restricted to habenula-target subnuclei, plus an increase in staining intensity of SP cells intrinsic to the IPN. There was no recovery of ChAT staining. A similar pattern of SP staining resulted following habenular transplants into neonatal hosts, but in addition there was a partial recovery of normal ChAT staining in cholinergic subnuclei and anomalous ChAT staining in normally peptidergic subnuclei. Control transplants of embryonic thalamus cells placed into adult hosts produced a surprising pattern of ChAT staining in the IPN identical to that seen with habenula transplants placed into neonatal hosts; the adult IPN was thus able to support reinnervation mediated by an aberrant cholinergic source while being refractory to its normal habenular cholinergic afferents. This pattern of results implies regulation by the IPN of habenular SP and cholinergic innervation, and some interaction between the maturing normal cholinergic afferents and their targets that is missing when these afferent sources are abnormal.

One of the enduring questions in developmental neurobiology is how the remarkable specificity in patterns of neural innervation of a target structure is achieved. Attempting to answer this question using in vivo experimental models has been difficult, particularly in the complex environment of the CNS. A recent approach that shows promise is the use of fetal transplants to investigate the mechanisms regulating innervation (Foster et al., 1985, 1988a,b; Freund et al., 1985; Gage and Bjorklund, 1986; Gibbs et al., 1986; Anderson et al., 1988; Radel et al., 1990; Sotelo and Alvarado-Mallart, 1991). In the most common of these paradigms, a target is denervated, and then transplants of homotopic or heterotopic cells are placed near the target and

\footnotetext{
Received Dec. 11, 1991; revised Mar. 9, 1992; accepted Mar. 25, 1992.

This work was supported by NIH Grants NS16556 (M.M.) and NS28856 (F.H.). We are grateful for the expert help provided by Amy DiCamillo and for the comments and discussions provided by Drs. Wendy Battisti, Sid Croul, and Alan Tessler.

Correspondence should be addressed to Marion Murray, Ph.D., Department of Anatomy and Neurobiology, The Medical College of Pennsylvania, 3200 Henry Avenue, Philadelphia, PA 19129.

Copyright (C) 1992 Society for Neuroscience $0270-6474 / 92 / 123272-11 \$ 05.00 / 0$
}

the patterns of reinnervation are examined. The value of this method depends to a great extent on the degree to which specific projections can be delineated in the modcl systcm choscn. For that reason, the habenulo-interpeduncular system, with its sharply compartmentalized pattern of normal innervation, seems an especially useful model for using transplants to investigate mechanisms responsible for afferent specificity.

The habenulo-interpeduncular system is simply organized. The paired medial habenulae project through the fasciculi retroflexi (FR) to terminate in the unpaired interpeduncular nucleus (IPN) at the base of the midbrain. The dorsal portion of the medial habenula contains peptidergic cells, identified immunocytochemically by antibodies to substance P (SP) (Hokfelt et al., 1975), which project bilaterally and in an overlapping fashion to the lateral and dorsal subnuclei in the peripheral portion of the IPN (Artymyshyn and Murray, 1985), supplying these subnuclei with an extremely dense concentration of SP. The ventral portion of the medial habenula contains cholinergic cells, identified by antibodies to $\mathrm{ChAT}$, which project bilaterally and in an overlapping fashion to the central and intermediate subnuclei in the central region of the IPN (Fckenrode et al., 1987), supplying these subnuclei with the highest concentrations of $\mathrm{ACh}$ in the brain (Kasa, 1986). The cholinergic and peptidergic targets of the medial habenulae therefore occupy contiguous but separate subnuclei in the IPN, the boundaries of which are readily identified histologically. Bilateral lesions of the FR eliminate all ChAT from the IPN and all SP from habenular target subnuclei (Artymyshyn and Murray, 1985; Eckenrode et al., 1987). There are other sources of SP in the IPN, located caudally in the central subnucleus and in the ventral sector of the rostral subnucleus, which provide the minor amounts of non-habenular SP staining remaining after FR lesions. These other sources do not project to the habenular SP targets, and they do not restore SP staining to habenular targets after FR lesions (Artymyshyn and Murray, 1985).

The normal development of the cholinergic and peptidergic habenular afferents to the IPN differs. The peptidergic and cholinergic habenular cells have closely overlapping birthdays [embryonic days 16-17 (E16-E17) and E17-E18, respectively; Lenn and Bayer, 1986], but the SP innervation is present in the IPN at birth, while ChAT staining cannot be demonstrated before the second postnatal week (Barr et al., 1987).

The projections to the IPN are characterized by robust lesion induced plasticity (Lenn, 1978; Lenn et al., 1979; Murray et al., 1979; Artymyshyn and Murray 1985; Barr et al., 1987; Battisti et al., 1987, 1989; Eckenrode et al., 1987), and this has been particularly well described for the habenular projections. Unilateral FR lesions in rats evoke partial replacement of SP, and this sprouting is similar whether the lesion is made in neonates or adults (Artymyshyn and Murray, 1985; Barr et al., 1987). 
Table 1. Summary of transplant characteristics and host IPN immunocytochemical staining

\begin{tabular}{|c|c|c|c|c|c|c|c|c|}
\hline \multirow[b]{2}{*}{ Animal } & \multirow[b]{2}{*}{ FR lesion } & \multirow[b]{2}{*}{ Lesion/TP age } & \multirow[b]{2}{*}{ Donor age } & \multirow[b]{2}{*}{ TP source } & \multirow[b]{2}{*}{ TP location } & \multicolumn{3}{|c|}{ IPN staining } \\
\hline & & & & & & SP (ros) & SP (lat) & ChAT \\
\hline 36 & $\mathrm{C}$ & $\mathrm{Ad} / \mathrm{Ad}$ & E15 & $\mathrm{Hb}$ & VTA & + & + & - \\
\hline 45 & I & $\mathrm{Ad} / \mathrm{Ad}$ & E14 & $\mathrm{Hb}$ & - & - & + & + \\
\hline 90 & $\mathrm{C}$ & $\mathrm{Ad} / \mathrm{Ad}$ & E15 & $\mathrm{Hb}$ & VTA & + & + & - \\
\hline 94 & $\mathrm{C}$ & $\mathrm{Ad} / \mathrm{Ad}$ & E14 & $\mathrm{Hb}$ & VTA & + & + & - \\
\hline 101 & $\mathrm{C}$ & $\mathrm{Ad} / \mathrm{Ad}$ & E14 & $\mathrm{Hb}$ & - & - & - & - \\
\hline 104 & $\mathrm{C}$ & $\mathrm{Ad} / \mathrm{Ad}$ & E15 & $\mathrm{Hb}$ & VTA & + & + & - \\
\hline 134 & $\mathrm{C}$ & $\mathrm{Ad} / \mathrm{Ad}$ & E14 & $\mathrm{Hb}$ & VTA & + & + & - \\
\hline 173 & $\mathrm{I}$ & $\mathrm{Ad} / \mathrm{Ad}$ & E14 & $\mathrm{Hb}$ & - & - & - & - \\
\hline 203 & $\mathrm{C}$ & $\mathrm{Ad} / \mathrm{Ad}$ & E15 & $\mathrm{Hb}$ & VTA & + & + & - \\
\hline 204 & $\mathrm{C}$ & $\mathrm{Ad} / \mathrm{Ad}$ & E15 & $\mathrm{Hb}$ & VTA & + & + & - \\
\hline 205 & $\mathrm{C}$ & $\mathrm{Ad} / \mathrm{Ad}$ & E15 & $\mathrm{Hb}$ & VTA & + & + & - \\
\hline FRK & $\mathrm{C}$ & $\mathrm{Ad} / \mathrm{Ad}$ & E18 & $\mathrm{Hb}$ & VTA & - & + & - \\
\hline 54 & $\mathrm{C}$ & P3/Ad & E18 & $\mathrm{Hb}$ & VTA & + & - & - \\
\hline Hb6 & $\mathrm{C}$ & $\mathrm{Ad} / \mathrm{Ad}$ & E15 & $\mathrm{Hb}$ & $\mathrm{dD}$ & - & + & - \\
\hline $\mathrm{Hb} 9$ & $\mathrm{C}$ & $\mathrm{Ad} / \mathrm{Ad}$ & E15 & $\mathrm{Hb}$ & dD & - & + & - \\
\hline 43 & $\mathrm{C}$ & P3/P6 & E15 & $\mathrm{Hb}$ & VTA & + & + & + \\
\hline 48 & I & $\mathrm{P} 3 / \mathrm{P} 6$ & E14 & $\mathrm{Hb}$ & - & - & + & + \\
\hline 49 & $\mathrm{C}$ & $\mathrm{P} 3 / \mathrm{P} 6$ & E14 & $\mathrm{Hb}$ & VTA & + & + & - \\
\hline 60 & $\mathrm{C}$ & P3/P10 & E14 & $\mathrm{Hb}$ & $\mathrm{CePe}$ & - & - & + \\
\hline 61 & $\mathrm{C}$ & P3/P10 & E15 & $\mathrm{Hb}$ & $\mathrm{CePe}$ & + & + & + \\
\hline 62 & $\mathrm{C}$ & $\mathrm{P} 3 / \mathrm{P} 10$ & E15 & $\mathrm{Hb}$ & VTA & + & + & + \\
\hline 64 & $\mathrm{C}$ & $\mathrm{P} 3 / \mathrm{P} 3$ & E15 & $\mathrm{Hb}$ & $\mathrm{CeT}$ & + & + & + \\
\hline 138 & $\mathrm{C}$ & $\mathrm{Ad} / \mathrm{Ad}$ & E14 & Th & VTA & + & + & + \\
\hline 139 & $\mathrm{C}$ & $\mathrm{Ad} / \mathrm{Ad}$ & E14 & Th & VTA & + & + & + \\
\hline 207 & $\mathrm{C}$ & P3/P6 & E15 & Th & SN & - & - & + \\
\hline 219 & $\mathrm{C}$ & $\mathrm{Ad} / \mathrm{Ad}$ & E15 & $\mathrm{Th}$ & $\mathrm{CeT}$ & + & - & + \\
\hline
\end{tabular}

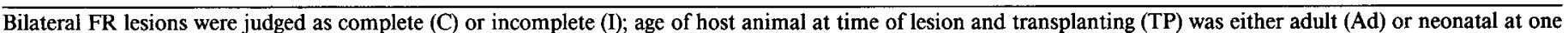

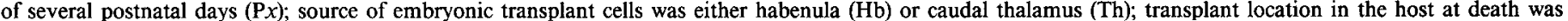

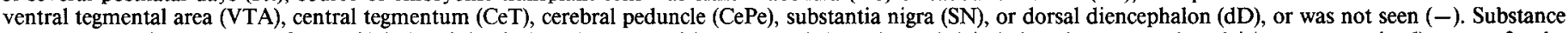

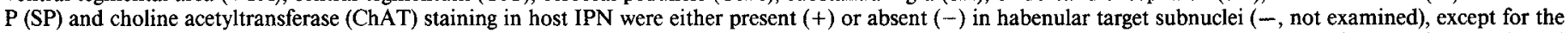

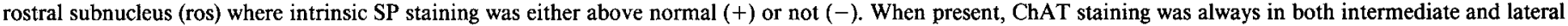
subnuclei, while SP staining was in lateral subnuclei (lat).

The same lesion will evoke replacement of the ChAT staining but only if the lesion is made in the neonate (Barr et al., 1987; Eckenrode et al., 1987). The cholinergic and peptidergic systems therefore differ in that the peptidergic system retains the capacity to sprout throughout life, while the cholinergic habenular cells manifest this ability only during a limited stage in their postnatal development, a period that coincides with the development of the cholinergic projection (Barr et al., 1987; Contestabile et al., 1990).

The developmental regulation of both normal innervation and reactive reinnervation could imply developmental changes in the IPN target cells or changes in the cholincrgic habcnular neurons that limit the ability of these cells to form afferent projections. One way to test these hypotheses is through use of transplants of fetal habenular neurons. If habenular cells lose their ability to sprout because of changes associated with their maturation, then fetal cells transplanted to adult animals should still retain competence to innervate mature denervated targets. If, on the other hand, it is the maturity of the target (IPN) that limits competence to accept innervation, then transplanted fetal habenular cells should be incapable of innervating adult host cells. Furthermore, the results of the sprouting experiments suggest that the cholinergic and peptidergic components of this system should behave differently. Since cholinergic habenula neurons do not sprout in the adult but peptidergic neurons do, transplanted cholinergic neurons would be expected to mediate reinnervation of the IPN only in neonatal hosts, while transplanted peptidergic neurons would be expected to produce recovery of IPN staining in both neonatal and adult hosts.

In the following study, we compared reinnervation of the IPN by transplanted embryonic habenular cells, following denervation by FR lesions in adult and neonatal hosts. As a control for nonspecific effects of transplanted fetal tissue, we also transplanted embryonic thalamus cells into other host animals with FR lesions, since in the rat, normal adult thalamus contains no intrinsic cholinergic (Jones, 1985; Hallanger et al., 1990) or peptidergic (Jonakeit et al., 1991) cells and the IPN is not a target for projections from thalamus under normal conditions.

\section{Materials and Methods}

\section{Surgery}

$F R$ lesions. Stereotaxic thermal lesions of the FR were made in deeply anesthetized 3-4-d-old female rat pups $(n=13)$ or adult female rats $(n$ $=20$ ), all of the Sprague-Dawley strain (Barr et al., 1987; Eckenrode et al., 1987). Pups were anesthetized by methoxyflurane inhalation, while adults were anesthetized using $4 \%$ chloral hydrate $(1 \mathrm{ml} / 100 \mathrm{gm}$ body weight). Of these lesioned animals, four pups and three adults served as lesion-only controls; all other animals received transplants of either embryonic habenula or thalamic cells. In three host animals, two adult and one neonate, the FR lesions were found to be incomplete; these animals served as post hoc controls for the contribution of a denervated target to successful survival of the transplanted cells. The roster of 


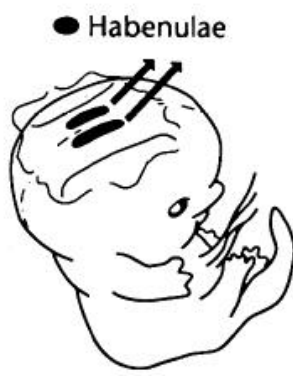

Figure 1. Diagram of transplant procedure. Habenulae are removed from fetuses, usually E14-E15, and cultured for several days. The cells are then dissociated and injected into the midbrain, usually near the denervated IPN. Hosts are then available for behavioral tests to determine functional significance of transplanted cells $(T P)$ (see following companion article, Haun et al., 1992).

E14/15

animals is listed in Table 1. Postoperative recovery was uneventful, and survival was virtually $100 \%$.

Transplant methods (Fig. 1). For all but two hosts, donor tissue was taken from Sprague-Dawley rat embryos at 14-15 d of gestation. In two cases, habenular tissue was removed from E18 embryos to serve as a control for the effect of greater maturity of the cells at time of harvesting. Dams were deeply anesthetized with $7 \%$ chloral hydrate $(0.5$ $\mathrm{ml} / 100 \mathrm{gm}$ body weight) and laparotomized, and the fetuses were removed individually and placed on a cold pack. For habenula dissection, the cortices were reflected, exposing the paired habenulae on the dorsal surface of the diencephalon, which were then excised under visual control. For the thalamus dissection, the diencephalic vesicle was exposed by a mid-sagittal cut through the brain, the hypothalamus was removed, and the ventral posterior quarter of the remaining diencephalon was excised (Fig. 2).

After meninges and attached blood vessels were removed, the habenula and thalamus pieces (each approximately $1 \mathrm{~mm} \times 0.5 \mathrm{~mm}$ ) were then cultured as explants (two pieces of one type per $35 \mathrm{~mm}$ Falcon

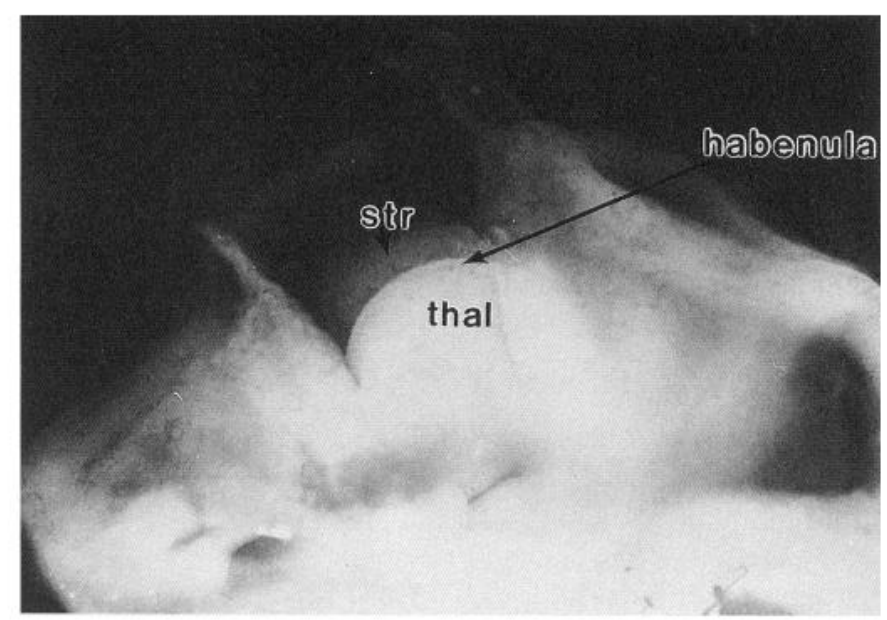

Figure 2. Mid-sagittal view of rat embryo late on E14 that shows location of habenular and posterior thalamic (thal) regions that are dissected for transplantation. With this dissection, the striatum (str) is distinct by its more lateral location and more rostral extent, compared to the diencephalon.
Primaria dish, 3-5 mm apart). Culture medium was a modified Ham's F-10, described in detail in Eagleson et al. (1990). The culture dishes were maintained at $33.5-34.5^{\circ} \mathrm{C}$ in a $90 \%$ air, $10 \% \mathrm{CO}_{2}$ high-humidity atmosphere for 4-5 d. At the end of this period, the tissue was enzymatically dissociated for $40 \mathrm{~min}$ at room temperature using a cocktail of $0.2 \%$ trypsin and $0.15 \%$ collagenase/dispase. Trypsin inhibition was accomplished by two 2 min washes in Ham's F-10 containing $15 \%$ porcine serum, $1 \mu \mathrm{M}$ EDTA, and $20 \mu \mathrm{g} / \mathrm{ml}$ DNase. The resulting cell suspension was then stored at $4^{\circ} \mathrm{C}$ in $\mathrm{F}-10$ containing $50 \mu \mathrm{g} / \mathrm{ml}$ heparan sulfate, $200 \mu \mathrm{g} / \mathrm{ml} \mathrm{GM1}$ ganglioside mixture, and $50 \mu \mathrm{M}$ hexacosonal. Prior to injecting this cell suspension into the host, an aliquot was assayed for cell viability and concentration using hemocytometer counts of trypan blue-excluding cells. Viability was never less than $87 \%$. The volume of cell suspension transplanted was adjusted so that approximately the same number of viable cells $\left(6-8 \times 10^{4}\right)$ was placed into each animal (Brundin et al., 1985). The injected volumes varied between $2 \mu \mathrm{l}$ and $6 \mu \mathrm{l}$, but there were no significant correlations between injection volume and either subsequent transplant volume or number of SP- or ChAT-positive neurons in the transplants.

Injections were made using a $10 \mu \mathrm{l}$ Hamilton syringe fitted with a specially prepared glass micropipette tip (OD, $0.45 \mathrm{~mm}$ ). In most cases the injections were aimed at the region of the ventral midbrain near the denervated IPN. In two cases, transplants were placed dorsally in the diencephalon, near the lesion in the vicinity of the habenula.

Most host rats had received a bilateral FR lesion either 7-10 d (adult host) or 2-6 d (neonatal host) prior to transplantation. The host rat was anesthetized and placed in the stereotaxic apparatus, and the needle was lowered into the brain according to predetermined coordinates localizing the degenerated FR pathway. The cells were injected slowly over 5-10 $\mathrm{min}$, the needle was removed, the wound sutured, and the rat allowed to recover before being returned to its home cage.

All animals were allowed to survive $2-3$ months posttransplantation before death.

\section{Anatomical methods}

At death, the animals were overdosed with chloral hydrate and perfused intracardially with normal saline, followed by $4 \%$ paraformaldehyde. Brains were removed and stored in $30 \%$ sucrose before sectioning.

Verification of lesion and location of transplant. The brains were blocked such that one block contained the FR lesion site and the other contained the IPN. The blocks were cryoprotected in $30 \%$ sucrose and frozen. Sections $(30 \mu \mathrm{m})$ were collected serially, and every fourth section was stained with cresyl violet to permit identification of the lesions and the transplant site. The lesion and the transplant are normally not present in the same sections, and the slides were coded so that the success of 

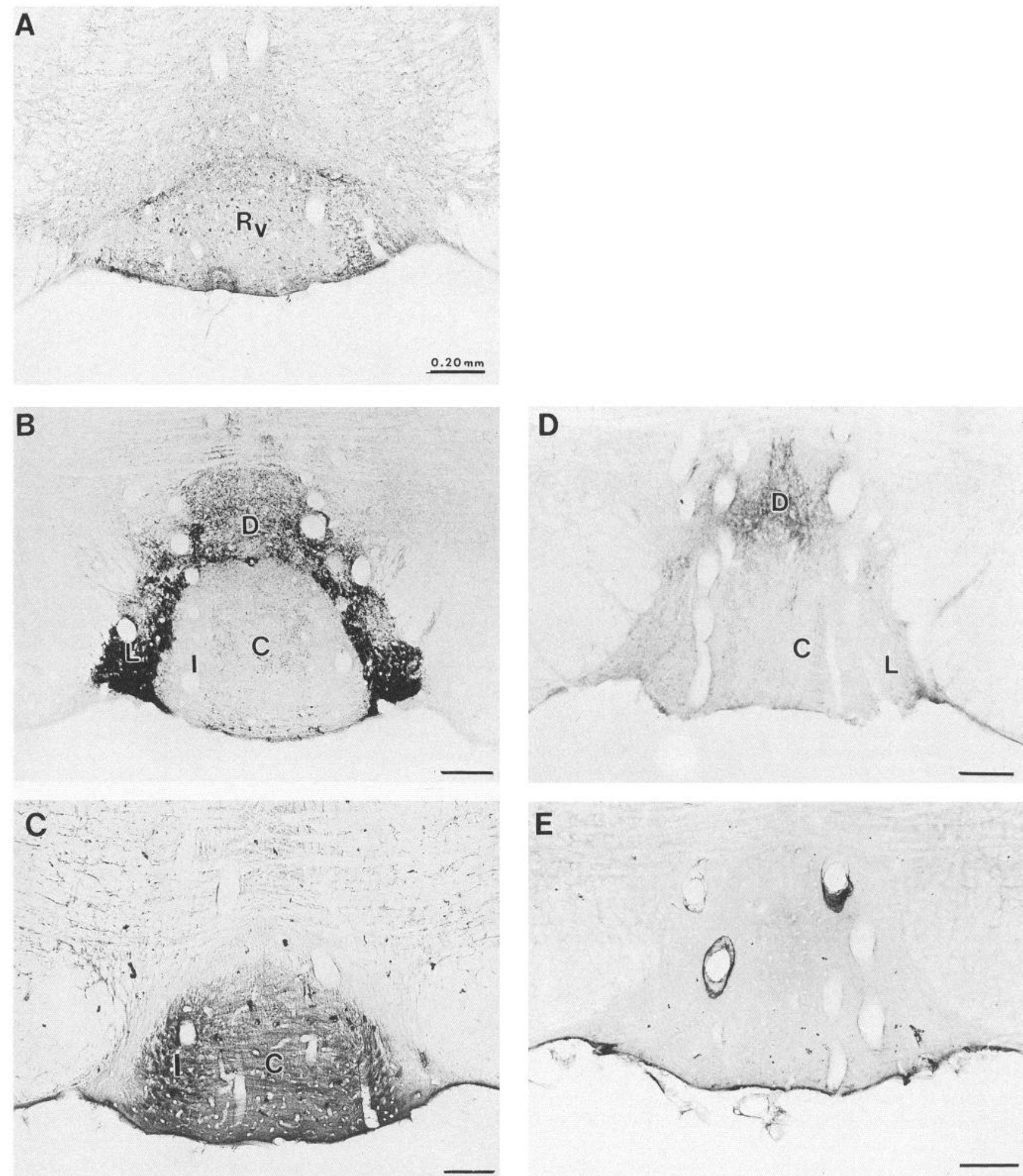

Figure 3. Staining patterns in the normal and FR-lesioned IPN. $A-C$, Control IPN. $D$ and $E$, IPN after bilateral FR lesion. $A$, Section stained for SP through rostral subnucleus of the IPN. Note population of cells in the ventral sector of the rostral subnucleus $\left(R_{v}\right)$. $B$, Section stained for SP through caudal IPN. Note dense staining in the lateral subnuclei $(L)$, somewhat less dense staining in the dorsal $(D)$ subnucleus, virtual absence of staining in intermediate subnuclei $(I)$ (identifiable by presence of blood vessels), and very faint staining in the central portion of the central (C) subnucleus. $C$, Section stained for ChAT through the caudal IPN. Note dense coarse staining in the intermediate subnuclei and dense staining characterized by horizontal pattern of axonal staining in the central subnucleus (Artymyshyn and Murray, 1985; Eckenrode et al., 1987). D, Section through caudal IPN showing SP staining after bilateral FR lesion. Note complete loss of staining in lateral subnuclei, but retention of moderate staining in dorsal subnucleus and very faint staining in central subnucleus. $E$, Section through caudal IPN stained for ChAT after bilateral FR lesion. Note complete loss of staining. 
Figure 4. Nissl-stained section showing denervated IPN and laterally a habenular transplant $(T P)$. Note well-integrated appearance of transplant and dense cellularity.

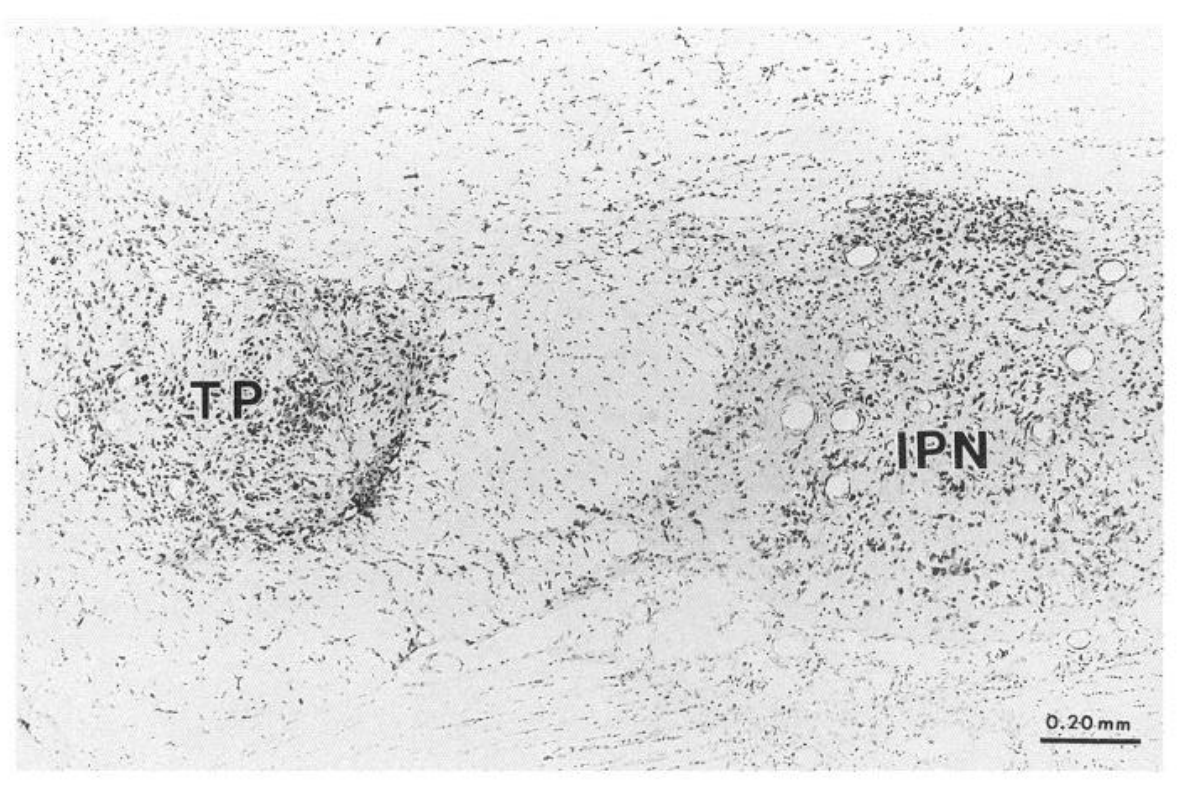

the lesion and the presence or absence of a transplant were determined without knowledge of the staining patterns in the IPN.

Immunocytochemistry. The remaining sections were processed for immunocytochemistry using antibodies to SP or ChAT (Artymyshyn and Murray, 1985; Eckenrode et al., 1987). Antibody to SP (Peninsula Labs. Inc.) was used at 1:10,000 dilution; the antibody to ChAT (Chemicon International, Inc.) was used at 1:3000 dilution. After two washes and $48 \mathrm{hr}$ of incubation in primary antibody at $4^{\circ} \mathrm{C}$, the sections were again washed and processed according to standard procedures for the Vectastain ABC kit (Vector Lab). To test for the specificity of the SP and ChAT antibodies, control sections were incubated with normal goat serum instead of primary antibodies and then processed as described above; in these cases, no specific staining was observed. Sections with IPN also contained substantia nigra, which stains densely for SP, and the oculomotor nucleus, which stains densely for ChAT. These two nuclei, which are independent of the medial habenulo-interpeduncular system, served as internal controls for immunocytochemical staining intensity in lesion-only and transplanted animals. The transplant was readily distinguished from host tissue by virtue of its relatively more dense neuronal population, compared to the surrounding host, and the frequent concentrations of glial cells and processes at the interface between host and transplant. The presence and characteristic aggregations of SP- and ChAT-stained cells in the transplant (see below) also served to distinguish the transplant from the host. To ensure that these stained cells were neurons, sections through the transplant were stained with antibodies against MAP2 and MAP1b (kindly supplied by Dr. I. Fischer, Department of Anatomy and Neurobiology, Medical College of Pennsylvania), which recognize neurons but not glia.

\section{Results}

\section{Normal habenulo-interpeduncular system}

The normal distribution of SP and ChAT in the intact IPN and the depletion of staining following bilateral FR lesions are shown in Figure 3. The FRs form two heavily myelinated bundles of axons, readily recognized as they exit from the ventral surface of the medial habenula, which course ventrally and caudally to enter the rostral pole of the IPN. Lesions placed in the caudal diencephalon destroy the FRs without damaging the IPN (see Murray et al., 1979, 1988).

\section{Transplants of habenular cells into adult FR-lesioned host}

Transplanted cells. Habenular cells transplanted into adult hosts that had undergone complete bilateral FR lesions survived well $(n=12)$. Most transplants were located near but not contiguous with the denervated IPN; distances calculated from serial sections ranged from $150 \mathrm{~mm}$ to $1740 \mathrm{~mm}$, with most transplants placed within $400-1200 \mathrm{~mm}$ from the IPN. In the two cases in which the transplants were placed near the ablated habenula rather than near the denervated IPN, the distances between transplant and target were 2220 and $2820 \mathrm{~mm}$. The transplanted cells were small, similar to normal habenular cells, and the transplant was usually well integrated, although some portion of the boundary was often demarcated by concentrations of glial cells and their processes (Fig. 4). Immunocytochemical staining revealed that SP- (Fig. $5 A$ ) and ChAT-stained cells (Fig. $5 B$ ) formed separate aggregates within a transplant, resembling in this respect the normal organization of the habenula (see also Lenn et al., 1990).

In two other adult animals the FR lesion was incomplete; in these cases no transplants were found, indicating that a denervated target is required for optimal survival of transplanted habenular neurons. In contrast, out of a total of 18 adult animals, prepared for this and a companion study, only one adult host with a complete bilateral FR lesion had no detectable surviving transplant.

$I P N$. The analysis of immunocytochemical staining in the IPN was unambiguous, as the FR lesions eliminate SP and ChAT from the habenular target subnuclei and there is normally very low background. Transplant-induced restoration of specific staining into FR-lesioned hosts was therefore readily apparent.

The habenular transplants into adult hosts did not restore cholinergic staining in the IPN, despite the presence of ChATpositive cells in the transplant. The same transplants did, however, mediate partial restoration of SP staining in the habenular target subnuclei in the IPN (Fig. 6A). Specific staining was seen in the lateral subnuclei, which were never stained in the FRlesioned control IPN in the absence of a habenular transplant. The extent of the restored staining was variable, although always less intense than normal. The staining was also usually symmetrical in the two lateral subnuclei even though the transplants were unilateral.

The transplants also mediated a consistent and very marked enhancement of staining of the intrinsic SP neurons and their processes in the rostral subnucleus (Fig. 6B). The staining as- 

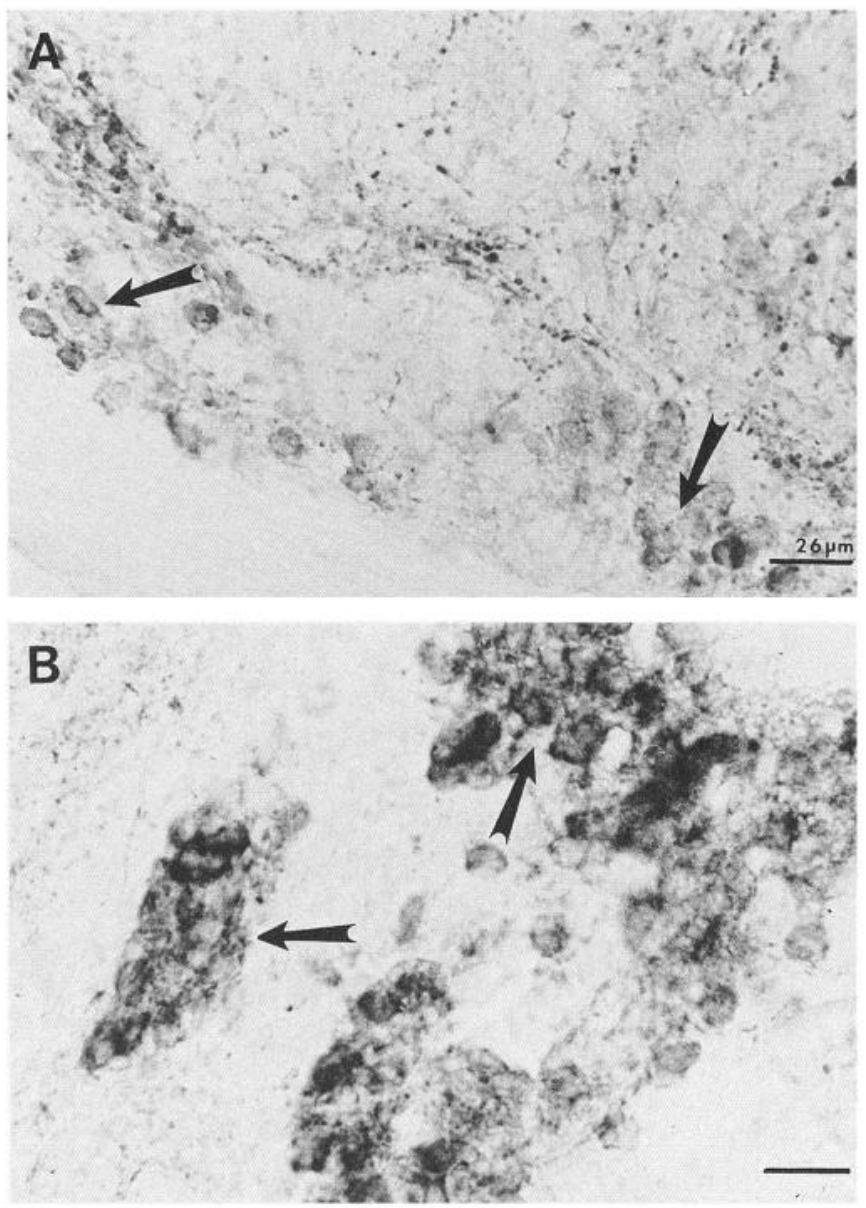

Figure 5. Habenular cells transplanted into adult hosts, stained immunocytochemically. $A$, Habenular transplant stained with SP. Note aggregates of small stained cells (arrows). $B$, Habenular transplant stained with ChAT. Note aggregates of small stained cells (arrows).

sociated with the intrinsic cells was restricted to its normal location in the ventral portion of the rostral subnucleus. Careful examination indicated no projection of these processes into the habenular SP target subnuclei, suggesting that these cells are not responsible for restoration of SP staining in the lateral subnuclei. There were only two examples of habenular transplants that did not enhance staining in this population of intrinsic cells, and these two transplants were those that had been placed dorsally within the diencephalon at a distance from the IPN that was much greater than that for our conventional transplants.

Since habenular SP neurons are born before habenular ChAT neurons (Lenn and Bayer, 1986), it seemed possible that the differences in the ability of SP and ChAT cells to restore staining might be due to the relatively greater maturity of the transplanted SP cells. We therefore examined two animals in which older fetal tissue (E18) was transplanted into adult hosts; in these transplants, the cholinergic cells would be more mature at the time of transplantation than those in the E14-E15 transplants used in the other experiments. The staining pattern, however, was similar; SP staining in habenular targets recovered partially, the intrinsic SP cells showed enhanced staining, but ChAT staining did not recover. The failure of cholinergic projections to form in adult host is therefore not due simply to a difference in maturity between implanted SP- and ChAT-containing habenular neurons.

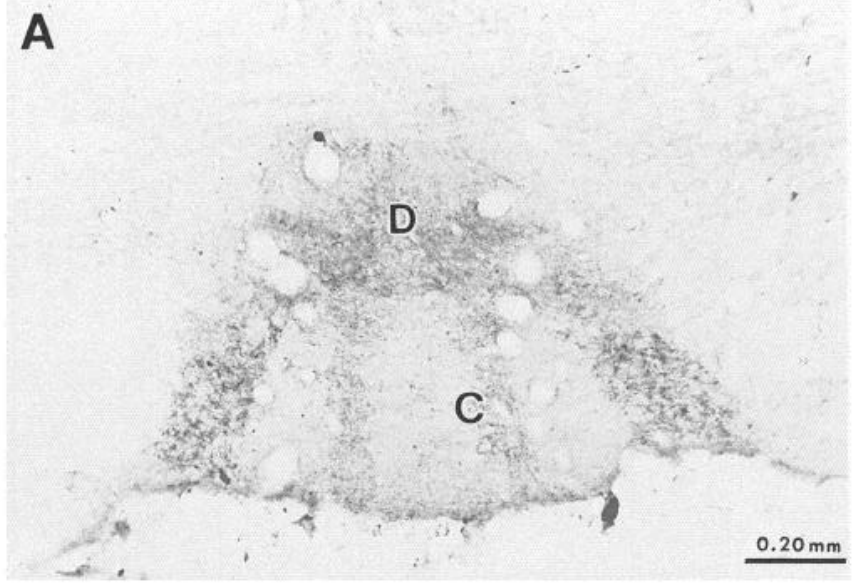

B

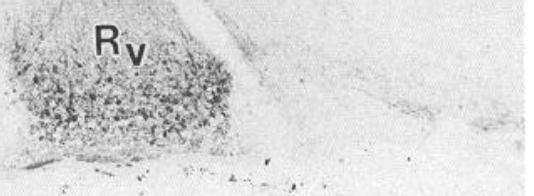

Figure 6. IPN in FR-lesioned adult host stained for SP. $A$, Caudal IPN showing restoration of staining in lateral subnuclei mediated by habenular transplant in adult. Note normal levels of non-habenular staining in the dorsal subnucleus $(D)$ and moderately enhanced levels of staining of non-habenular SP in the central subnucleus (C). B, Rostal IPN showing greatly enhanced staining of intrinsic SP cells in ventral sector of rostral subnucleus $\left(R_{v}\right)$.

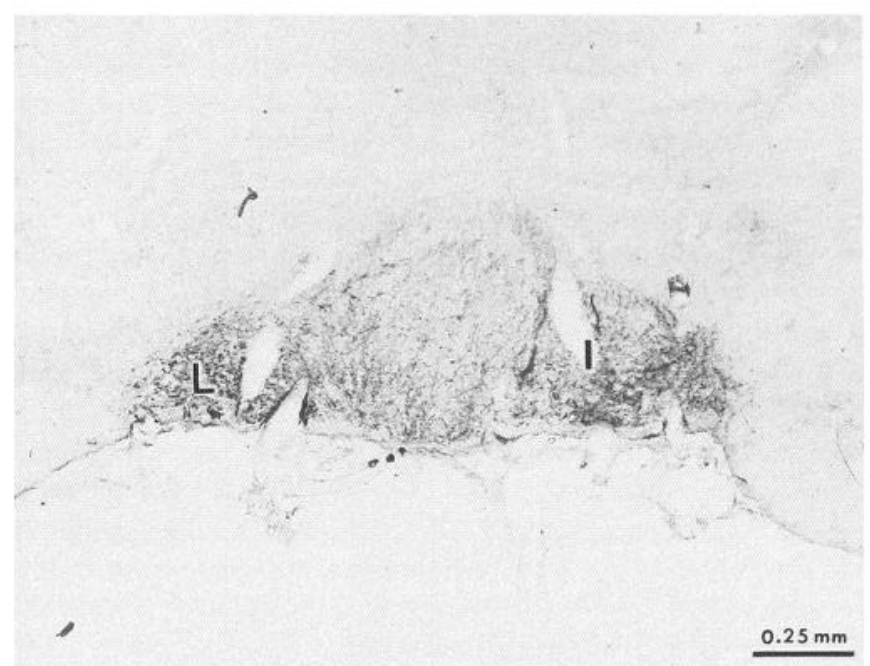

Figure 7. Caudal IPN showing restoration of ChAT staining in IPN mediated by neonatal habenular transplant. Note staining in intermediate subnuclei, around blood vessels at base of IPN. Note also that staining extends into lateral subnuclei, which never contain ChAT staining in intact or bilateral FR-lesioned IPN. 
Figure 8. Cholinergic staining of thalamic cells. $A$, Nissl-stained section through midbrain just rostral to adult FR-lesioned IPN showing transplant of thalamic cells $(T P)$. Note that thalamic transplant is well integrated and densely cellular. $B$, Section through thalamic transplant stained with ChAT. Note cells with robust processes. Transplanted thalamic cells are less well aggregated, larger and possess more prominent processes than transplanted habenular cells. $C$, Section through thalamus of E14 fetus, stained with ChAT, showing several ChAT-stained cells in thalamus at this stage of development (arrow). $D$, Higher magnification of field shown in $C$ to show neuronal morphology of stained cells (arrow).
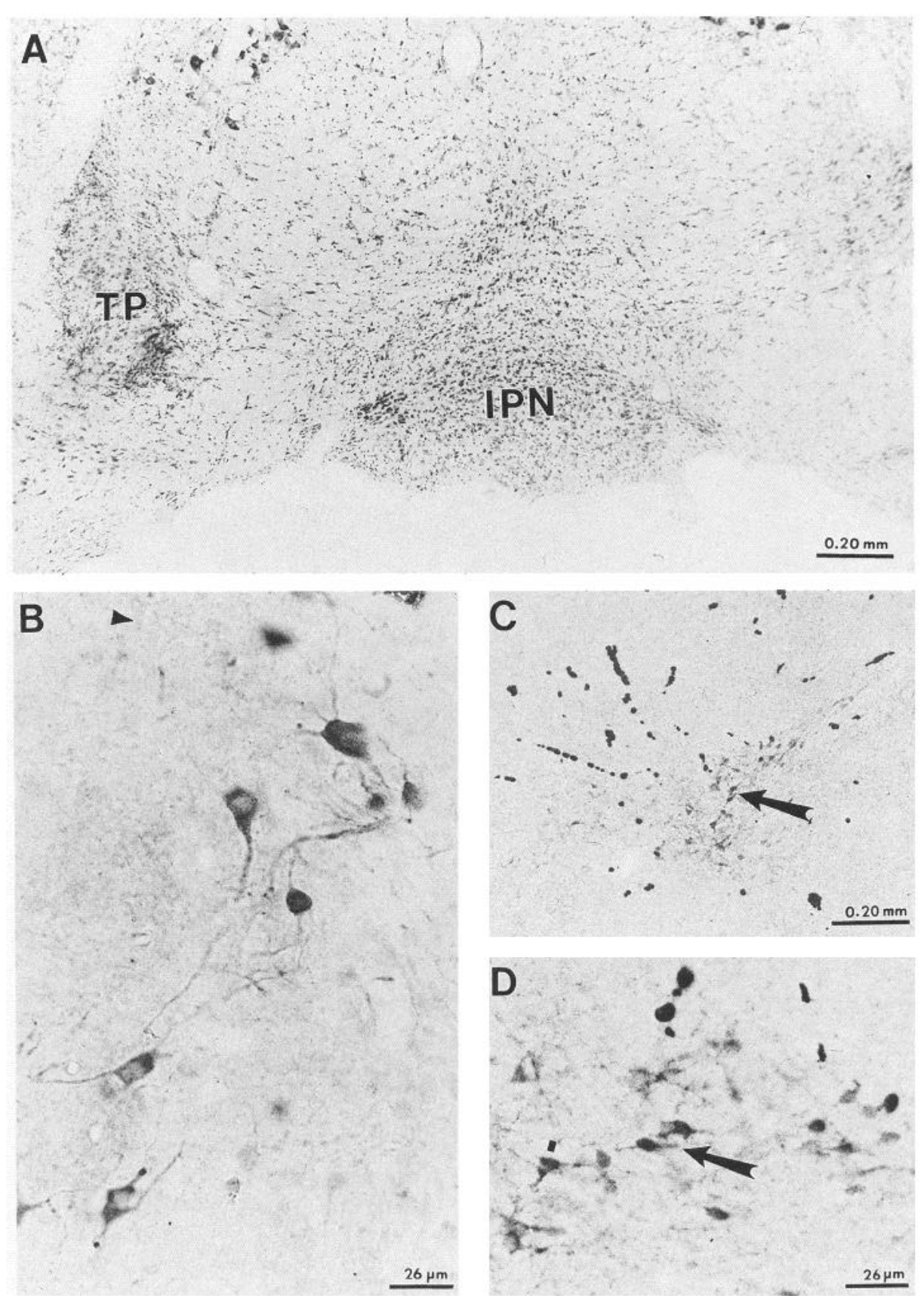

\section{Transplants of habenular cells into neonatal FR-lesioned host}

The habenular cells transplanted into neonatal-lesioned hosts were also well integrated and contained aggregates of SP- and ChAT-positive cells in cases in which the FR lesion was complete $(n=6)$. In one other case, habenular cells were placed in a neonatal host in which the FR lesion was incomplete; as in the cases of adult hosts with incomplete lesions, no transplant was seen.

In animals with successful neonatal lesions and transplants, ChAT staining was partially restored in the IPN (Fig. 7). Staining was more pronounced in the intermediate subnuclei than in the central subnucleus, and staining of the characteristic pattern of fibers that recross through the central subnucleus (see Ramon y Cajal, 1955) was never seen. Interestingly, ChAT staining was also found in the lateral subnuclei, where it is normally never found. This staining pattern therefore represents an aberrant projection of cholinergic afferents to a normally non-cholinergic target.

SP staining in the lateral subnuclei was also partially restored, but recovery of staining was similar to that seen after transplantation into adult hosts. Staining of the intrinsic cells was enhanced by the transplants and also to an extent similar to that seen in adult hosts.

\section{Transplants of thalamic cells into FR-lesioned host}

Since adult thalamus contains neither SP- nor ChAT-stained cell bodies, we used transplants of fetal thalamic tissue into FRlesioned host brains to serve as a control for nonspecific grafting effects $(n=4)$. These transplants also integrated well (Fig. $8 A$ ). 


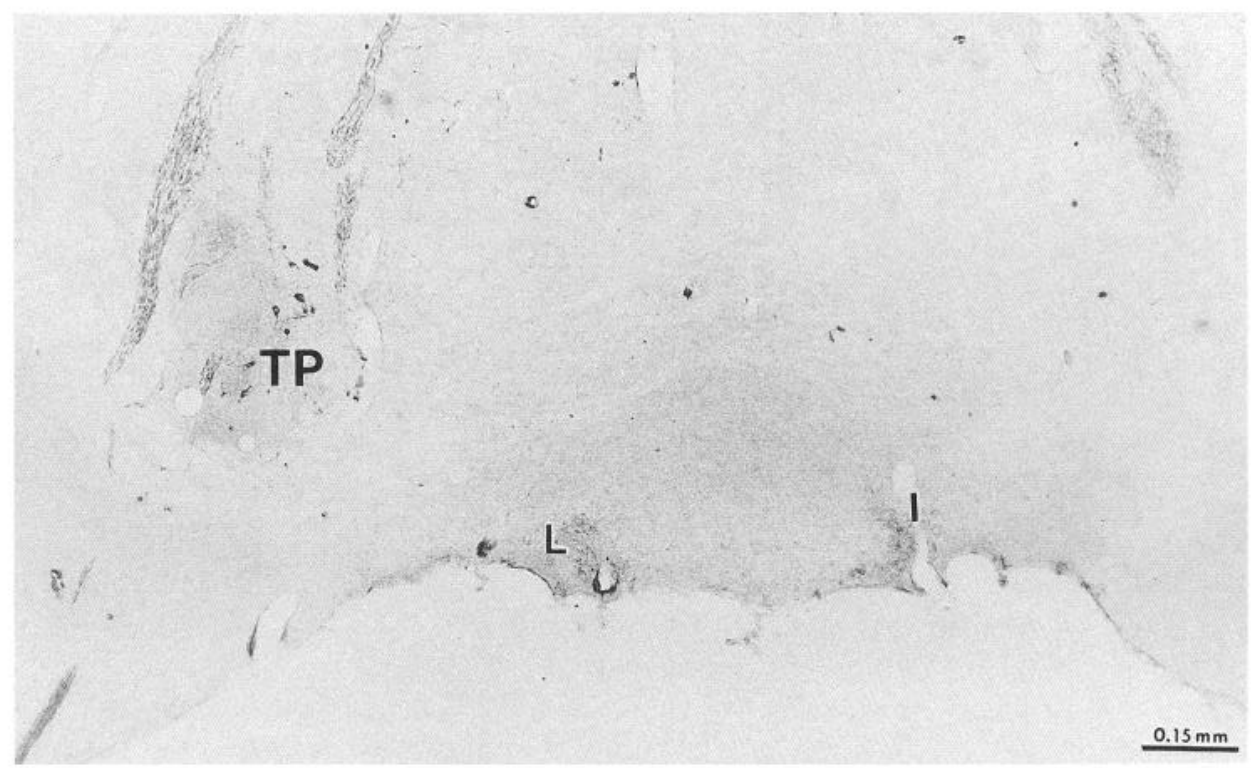

Figure 9. ChAT staining in FR-lesioned adult IPN mediated by thalamic transplant. Note transplant $(T P)$ containing several ChAT-stained neurons lying lateral to the IPN between fascicles of oculomotor axons. Note ChAT staining at the base of the IPN in the intermediate subnuclei, around the blood vessels, and extending into the lateral subnucleus ipsilateral to the transplant.
SP immunocytochemistry showed rare SP-positive neurons and small amounts of punctate SP staining in the transplant. The staining pattern for SP in the IPN was variable; two of the four hosts showed weak SP staining in the lateral subnuclei, and three showed extremely faint staining in the rostral subnucleus.

Surprisingly, many ChAT-positive neurons were found in all thalamic transplants (Fig. $8 B$ ). Immunocytochemical staining of E14 brains revealed the presence of a population of ChATpositive neurons that are not present in the postnatal thalamus (Fig. $8 C, D$ ). Immunocytochemistry of dissected pieces of E14 ventral posterior thalamus also revealed some ChAT-positive cells immediately after being placed in culture and considerably more ChAT-positive cells after $5 \mathrm{~d}$ in culture, the time at which the cells would be implanted into the hosts. The greater number of ChAT-positive cells seen after $5 \mathrm{~d}$ in culture may reflect preferential survival or induced expression of the cholinergic phenotype by some agent(s) in the culture medium. Transplantation of these cells to the FR denervated host therefore appears to have the unexpected effect of supporting the survival or continued expression of ChAT in this population of neurons. These thalamic transplants containing ChAT-positive neurons also supported the restoration of ChAT staining in the IPN (Fig. 9). The restored staining was densest in the intermediate subnucleus, was often symmetrical, and sometimes extended into the lateral subnuclei, the habenular SP targets; the staining pattern mediated by thalamic cells therefore resembles the staining pattern mediated by habenular ChAT neurons implanted into neonatal hosts.

\section{Discussion}

The major findings of the present study are that fetal habenular neurons survive when transplanted into the parenchyma of neonatal or adult hosts whose FRs have been sectioned bilaterally, and that these transplanted neurons can mediate restoration of specific transmitter staining patterns in the IPN. The restoration of specific staining is most readily explained by reinnervation of target cells by the transplanted neurons, but proof of this awaits electron microscopic verification of the formation of synaptic contacts. The successful survival of our transplanted tissue appeared to be correlated with the denervation of the host target, the IPN, and may have also been related to using young (E14E15) donor tissue (N. J. Lenn, personal communication) plus a lesion/delay paradigm. A previous study using habenular transplants from older fetuses into hosts without FR lesions showed less successful survival (Lenn et al., 1990).

SP staining recovered partially in the habenular SP target subnuclei, and in addition, the staining of the intrinsic SP containing cells in the ventral sector of the rostral subnucleus of the IPN was enhanced. The pattern of SP staining in habenular SP targets mediated by transplanted habenular cells was similar to normal; the degree of recovered SP staining in habenular targets was always less than normal, but staining of the intrinsic system was much greater than normal. The ease with which staining is restored by host sprouting or by reinnervation with transplanted neurons in both adult and neonatal hosts indicates that the denervated target cells remain receptive to appropriate innervation.

ChAT staining also recovered partially when habenular transplants were placed into neonatal brains but not into adult brains. Surprisingly, ChAT staining also recovered when cell suspensions of fetal thalamus containing cholinergic cells were implanted into adult brains. ChAT staining mediated by transplants often extended into subnuclei normally never innervated by cholinergic systems, for example, the lateral subnuclei.

The pattern of restored ChAT staining shows interesting differences from the pattern of restored SP. There is clear evidence of restoration of ChAT staining within normal targets, the intermediate subnuclei, but also of extension into the lateral subnuclei, which are normally innervated by habenular SP neurons. This extension of ChAT staining into an aberrant target occurs with both habenular cell transplants, into neonatal hosts, and thalamic cell transplants, into adult hosts. This finding indicates that the neurons in the lateral subnuclei retain the capability of accepting cholinergic innervation throughout life even though they normally do not receive such innervation. The habenular cholinergic projection is nicotinic, and there are nicotinic receptors in the IPN (Swanson et al., 1987; Mulle et al., 1991); the lateral subnuclei, however, contain a high density of mus- 
carinic receptors (Rotter and Jacobowitz, 1984). It is tempting to suggest that the ability of the neurons in the lateral subnuclei to receive cholinergic innervation may be related to the presence of these high concentrations of muscarinic cholinergic receptors.

\section{Source of recovered staining}

We attribute the recovered staining to cholinergic and peptidergic neurons contained within the transplant. In the case of the cholinergic system, the recovered staining is most likely the result of direct innervation by cholinergic cells in the transplant, since there is no other source of ChAT within the IPN or near the IPN and there is no recovery of ChAT staining after FR lesions in the absence of transplanted fetal tissue containing cholinergic cells.

In the case of SP staining, the transplant may have both direct and indirect effects on staining in the IPN. The presence of intrinsic SP cells and the sparing of some SP staining after FR lesions raise the possibility that these non-habenular sources contribute to the restoration of staining. In fact, the most robust effect of the habenular transplants, placed either in adult or neonatal hosts, is the greatly enhanced staining of the intrinsic SP-containing cells in the ventral sector of the rostral subnucleus. The effect of habenular transplants on these cells appears to be specific since enhanced staining of the intrinsic SP system is not seen after thalamic transplants. These cells are therefore the obvious candidates for restoration of SP staining in the habenular targets. The intrinsic SP cells, however, do not provide SP staining to the habenular targets after FR lesions alone. The boundaries of the area occupied by these cells appear to be strictly maintained following lesions and transplants; the staining pattern therefore does not support the suggestion that these cells restore SP staining in the lateral subnucleus. The specificity of the effect suggests that enhancement of staining of these cells may be due to diffusible factors arising from those transplants that contain SP cells. This interpretation is supported by the two cases in which the transplanted habenular tissue was placed in the dorsal diencephalon, at a much greater distance from the denervated IPN than in our other hosts. In these cases, SP staining recovered in the habenular targets of the IPN but was not enhanced in the intrinsic cells.

\section{Transmitter-specific regulation of reinnervation}

Most studies in which fetal transplants have been used to reinnervate denervated structures have emphasized the restoration of normal patterns of projections and therefore, by extension, of normal innervation (Foster et al., 1985, 1988a,b; Freund et al., 1985; Zhou et al., 1985; Gage and Bjorklund, 1986; Gibbs et al., 1986; Anderson et al., 1988; Clarke et al., 1990; Radel et al., 1990). Transmitter-specific reinnervation often takes place regardless of whether the neurons are appropriate or inappropriate (but see Ikegami et al., 1991). For example, cholinergic staining in the hippocampus is restored by implanted septal neurons, the normal source, and also by implanted habenular or striatal neurons, abnormal sources of cholinergic input (Gibbs et al., 1986; Anderson et al., 1988; Clarke et al., 1990). The pattern of host target innervation by these heterologous transplants, however, may be abnormal (Nilsson et al., 1988). The mechanisms by which transmitter-specific pathways are reestablished would seem to implicate receptor changes in the target neurons that act in some cases to favor reestablishment of normal projections.

The pattern of restoration of SP staining shows a recovery limited to normal target areas and thus supports a transmitterspecific regulation of innervation mediated by the transplanted cells. The cholinergic staining, however, invades abnormal target areas normally occupied by the SP habenular projection. SP is known to inhibit nicotinic cholinergic function (Stallcup and Patrick, 1980; Boyd and Leeman, 1987). The SP system develops before the cholinergic innervation (Barr et al., 1987), and its occupation of the lateral subnuclei may normally limit cholinergic innervation; the disruption of the temporal order of innervation created by FR lesions and habenular transplants may eliminate the advantage normally enjoyed by the SP cells.

Our finding of similar patterns of ChAT innervation mediated by appropriate habenular neurons and inappropriate thalamic neurons is generally in agreement with the results of others (Foster et al., 1985, 1988a; Gage and Bjorklund, 1986; Gibbs et al., 1986; Anderson et al., 1988). What was surprising was that thalamic but not habenular cholinergic neurons could support restoration of ChAT staining in the adult IPN. Our hypothesis, based on both normal development and lesion-induced sprouting in the habenular cholinergic system, suggested a temporal limitation on innervation. This limitation could be due to changes in the target, for example, cessation of release of adequate amounts of trophic substances, or to changes in the afferents, for example, cessation of synthesis of adequate types or numbers of receptors for the trophic substances. The evidence of innervation of adult host by fetal thalamic cholinergic neurons indicates that the host target cells remain receptive; the parallel failure of fetal habenular cells to innervate adult host cholinergic targets, while successfully innervating the same targets in neonatal hosts, suggests that innervation is not dependent on features associated with the immature afferents. Furthermore, Gibbs et al. (1986) and Anderson et al. (1988) have shown that fetal habenular cells transplanted into adult hosts can restore normal staining patterns to denervated hippocampus, an anomalous target. Habenular neurons thus retain the capability to form new projections even in an adult and in foreign territory.

Our hypothesis therefore did not fully account for our results. Additional factors must be involved in the establishment of the habenulo-interpeduncular cholinergic projection. The habenular cholinergic projection does have the peculiar feature of innervating the system of crest synapses that densely populate the intermediate subnuclei. These synapses are complex structures, in which one terminal arises from cells in the left habenula and the other terminal arises from cells in the right habenula (Lenn, 1976; Murray et al., 1979; Lenn et al., 1983). This left-right organization develops postnatally and indicates an additional constraint on the formation of synaptic complexes involving habenular neurons that may not operate in the formation of synapses by non-habenular cholinergic neurons.

Another possibility is that the transplanted thalamic neurons retain immature characteristics whereas habenular neurons transplanted near their normal target do not. Immature transmitter phenotypes have been shown to be retained and possibly enhanced in transplanted cortical cells, whether grafted into homotopic or heterotopic sites (Park et al., 1986; Herman et al., 1988). It is possible that immature phenotypes are retained especially when transplanted cells are placed in an aberrant location, while normal development may occur if the ectopically transplanted cells are able to innervate their normal targets. When transplants of habenular, instead of thalamic, cells are placed near the denervated IPN, the "price paid" by the habenular cholinergic neurons for innervating an appropriate tar- 
get is being thereby subject to the normal constraints regulating IPN innervation, whereas the less mature thalamic cholinergic neurons are also less responsive to these constraints.

\section{References}

Anderson KJ, Gibbs RB, Cotman CW (1988) Transmitter phenotype is a major determinant in the specificity of synapses formed by cholinergic neurons transplanted to the hippocampus. Neuroscience 25: 19-25.

Artymyshyn R, Murray M (1985) Substance $P$ in the interpeduncular nucleus of the rat: normal distribution and effects of deafferentation. J Comp Neurol 231:78-90.

Barr GA, Eckenrode TC, Murray M (1987) Normal development and the effects of early deafferentation in choline acetyltransferase, substance $\mathrm{P}$ and serotonin like immunoreactivity in the interpeduncular nucleus. Brain Res 418:301-313.

Battisti WP, Artymyshyn R, Murray M (1987) Norepinephrine in the interpeduncular nucleus of the rat: normal distribution and the effects of deafferentation. Brain Res 418:287-300.

Battisti WP, Artymyshyn R, Murray M (1989) $\beta_{1}$ and $\beta_{2}$ adrenergic ${ }^{125}$ I-pindolol binding sites in the interpeduncular nucleus of the rat: normal distribution and the effects of deafferentation. $\mathbf{J}$ Neurosci 9 : 2509-2518.

Boyd ND, Leeman SE (1987) Multiple actions of substance $P$ that regulate the functional properties of acetylcholine receptors of clonal rat PC12 cells. J Physiol (Lond) 389:69-79.

Bregman BS, Goldberger ME (1982) Anatomical plasticity and sparing of function after spinal damage in the neonatal cat. Science 217:553555.

Brundin P, Isacson O, Bjorklund A (1985) Monitoring of cell viability in suspensions of embryonic CNS tissue and its use as a criterion for intracerebral graft survival. Brain Res 331:251-259.

Clarke DJ, Nilsson OG, Brundin P, Bjorklund A (1990) Synaptic connections formed by grafts of different types of cholinergic neurons in the host hippocampus. Exp Neurol 107:11-22.

Contestabile A, Virgili M, Barnabei O (1990) Developmental profiles of cholinergic activity in the habenulae and IPN of the rat. Int J Dev Neurosci 8:561-564.

Eagleson KL, Haun F, Cunningham TJ (1990) Different populations of dorsal lateral geniculate nucleus neurons have concentration-specific requirements for a cortically derived neuron survival factor. Exp Neurol 110:284-290.

Eckenrode TC, Barr G, Battisti W, Murray M (1987) Acetylcholine in the interpeduncular nucleus of the rat: normal distribution and effects of deafferentation. Brain Res 418:273-286.

Foster GA, Schultzberg M, Gage FH, Bjorklund A, Hokfelt T, Nornes H, Cuello AC, Verhofstad AAJ, Visser TJ (1985) Transmitter expression and morphological development of embryonic medullary and mesencephalic raphe neuroncs aftcr transplantation to the adult rat central nervous system. I. Grafts to the spinal cord. Exp Brain Res 60:427-444.

Foster GA, Schultzberg M, Gage FH, Bjorklund A, Hokfelt T, Cuello AC, Verhofstad AAJ, Visser TJ, Emson PC (1988a) Transmitter expression and morphological development of embryonic medullary and mesencephalic raphe neurones after transplantation to the adult rat central nervous system. II. Grafts to the hippocampus. Exp Brain Res 70:225-241.

Foster GA, Schultzberg M, Gage FH, Bjorklund A, Hokfelt T, Cuello AC, Verhofstad AAJ, Visser TJ, Emson PC (1988b) Transmitter expression and morphological development of embryonic medullary and mesencephalic raphe neurones after transplantation to the adult rat central nervous system. III. Grafts to the striatum. Exp Brain Res $70: 242-255$.

Freund TF, Bolam JP, Bjorklund A, Stenevi U, Dunnett SB, Powell JF, Smith AD (1985) Efferent synaptic connections of grafted dopaminergic neurons reinnervating the host neostriatum: a tyrosine hydroxylase immunocytochemical study. J Neurosci 5:603-616.

Gage FH, Bjorklund A (1986) Enhanced graft survival in the hippocampus following selective denervation. Neuroscience 17:89-98.

Gibbs RB, Anderson KJ, Cotman CW (1986) Factors affecting innervation in the CNS: comparison of three cholinergic cell types transplanted to the hippocampus of the rat. Brain Res 383:362-366.

Hallanger AE, Price SD, Lee HJ, Steininger TL, Wainer BH (1990)
Ultrastructure of cholinergic synaptic terminals in the thalamic anteroventral, ventroposterior and dorsal lateral geniculate nuclei of the rat. J Comp Neurol 299:482-492.

Haun F, Eckenrode TC, Murray M (1992) Habenula and thalamus cell transplants restore normal sleep behaviors disrupted by denervation of the interpeduncular nucleus. J Neurosci 12:3285-3293.

Herman JP, Abous N, Vigny A, Dulluc J, LeMoal M (1988) Distorted development of rat cerebral grafts. Dev Brain Res 40:81-88.

Hokfelt T, Kellerth JO, Nilsson G, Pernow B (1975) Substance P localization in the central nervous system and in some primary sensory neurons. Science 190:889-890.

Ikegami S, Nihonmatsu I, Kawamura H (1991) Transplantation of ventral forebrain cholinergic neurons to the hippocampus ameliorates impairment of radial-arm maze learning in rats with AF64A treatment. Brain Res 548:187-195.

Jonakeit GM, Ni L, Walker PD, Hart RP (1991) Development of substance $P$ (SP)-containing cells in the central nervous system: consequences of neurotransmitter co-localization. Prog Neurobiol 36:121.

Jones EG (1985) The thalamus. New York: Plenum.

Kasa $P$ (1986) The cholinergic systems in brain and spinal cord. Prog Neurobiol 26:211-227.

Lenn NJ (1976) Synapses in the interpeduncular nucleus: electron microscopy of normal and habenula lesioned rats. J Comp Neurol 166:73-100.

Lenn NJ (1978) Effect of neonatal deafferentation on synaptogenesis in the rat interpeduncular nucleus. J Comp Neurol 181:92-1 16.

Lenn NJ, Bayer SA (1986) Neurogenesis in the subnuclei of the rat interpeduncular nucleus and medial habenula. Brain Res Bull 16:219224.

Lenn NJ, Wong V, Hamill G (1979) Quantitative demonstration of somatic sprouting following dendritic deafferentation in the neonatal rat interpeduncular nucleus. Brain Res Bull 4:843-848.

I enn NJ, Wong V, Hamill G (1983) Left-right pairing at the crest synapses of rat IPN. Neuroscience 9:383-389.

Lenn NJ, Seeley PJ, Field PM, Raisman G (1990) Fetal medial habenular transplants: innervation of the rat interpeduncular nucleus. J Neural Transpl Plast 1:57-62.

Mulle C, Vidal C, Benoit P, Changeux JP (1991) Existence of different subtypes of nicotinic acetylcholine receptors in the rat habenulo-interpeduncular system. J Neurosci 11:2588-2597.

Murray M, Zimmer J, Raisman G (1979) Reinnervation in the rat interpeduncular nucleus after lesions of the fasciculus retroflexus. J Comp Neurol 186:34-51.

Murray M, Saffroy M, Torrens Y, Beaujovan JC, Glowinski J (1988) Tachykinin binding sites in the interpeduncular nucleus of the rat. Brain Res 459:76-92.

Nilsson OG, Clarke DJ, Brundin P, Bjorklund A (1988) Comparison of growth and reinnervation properties of cholinergic neurons from different brain regions grafted to the hippocampus. J Comp Neurol 268:204-222.

Park JK, Joh TH, Ebner FH (1986) Tyrosine hydroxylase is expressed by neocortical neurons after transplantation. Proc Natl Acad Sci USA 83:7495-7498.

Radel JD, Hankin MH, Lund RD (1990) Proximity as a factor in the innervation of host brain regions by retinal transplants. J Comp Neurol 300:211-229.

Ramon y Cajal S (1955) Histologie du systeme nerveux de l'homme et des vertebres, Vol II, pp 270-275. Madrid: Instituto Ramon y Cajal.

Rotter A, Jacobowitz DM (1984) Localization of substance P, acetylcholinesterase muscarinic receptors and alpha-bungarotoxin in the rat interpeduncular nucleus. Brain Res Bull 12:83-94.

Sotelo C, Alvarado-Mallart RM (1991) The reconstruction of cerebellar circuits. Trends Neurosci 14:350-357.

Stallcup WB, Patrick J (1980) Substance P enhances cholinergic receptor desensitization in a clonal nerve cell line. Proc Natl Acad Sci USA 77:634-638.

Swanson LW, Simmonds DM, Whiting PJ, Lindstrom J (1987) Immunohistochemical localization of neuronal nicotinic receptors in the rodent CNS. J Neurosci 7:3334-3342.

Zhou C-F, Raisman G, Morris RJ (1985) Specific patterns of fibre outgrowth from transplants to host mice hippocampi, shown immunohistochemically by the use of allelic forms of thy- 1 . Neuroscience 16:819-833. 